\title{
Cell-Type Specific Phase Precession in Layer II of the Medial Entorhinal Cortex
}

\author{
Eric T. Reifenstein, ${ }^{1,2}$ CChristian L. Ebbesen, ${ }^{2,3}$ Qiusong Tang, ${ }^{2}$ Michael Brecht, ${ }^{2}$ Susanne Schreiber, ${ }^{1,2}$ \\ and ${ }^{-R i c h a r d ~ K e m p t e r ~}{ }^{1,2}$ \\ ${ }^{1}$ Institute for Theoretical Biology, Department of Biology, Humboldt-Universität zu Berlin, 10115 Berlin, Germany, ${ }^{2}$ Bernstein Center for Computational \\ Neuroscience Berlin, 10115 Berlin, Germany, and ${ }^{3}$ Berlin School of Mind and Brain, Humboldt-Universität zu Berlin, 10099 Berlin, Germany
}

The identity of phase-precessing cells in the entorhinal cortex is unknown. Here, we used a classifier derived from cell-attached recordings to separate putative pyramidal cells and putative stellate cells recorded extracellularly in layer II of the medial entorhinal cortex in rats. Using a novel method to identify single runs as temporal periods of elevated spiking activity, we find that both cell types show phase precession but putative stellate cells show steeper slopes of phase precession and larger phase ranges. As the two classes of cells have different projection patterns, phase precession is differentially passed on to different subregions of the hippocampal formation.

Key words: entorhinal cortex; hippocampus; phase precession; pyramidal cell; stellate cell; temporal code

\section{Significance Statement}

It is a great challenge for neuroscience to reveal the cellular basis of cognitive functions. One such function is the ability to learn and recollect temporal sequences of events. The representation of sequences in the brain is thought to require temporally structured activity of nerve cells. How different types of neurons generate temporally structured activity is currently unknown. In the present study, we use a computational classification procedure to separate different cell types and find that a subpopulation of cells, so-called stellate neurons, exhibits clear temporal coding. Contrary to the stellate cells, pyramidal cells show weaker temporal coding. This discovery sheds light on the cellular basis of temporal coding in the brain.

\section{Introduction}

Large-scale oscillations play an important role in many parts of the brain. They may functionally couple different brain regions and provide the basis for temporal coding (Buzsáki and Draguhn, 2004; O'Keefe and Burgess, 2005). Throughout the entorhinalhippocampal network, large-amplitude theta oscillations (6-11 $\mathrm{Hz}$ ) can be observed in the local field potential. Different cell types show different degrees of locking to the local theta oscillations (Klausberger and Somogyi, 2008; Mizuseki et al., 2009), and principal cells shift their firing phases consistently from late to

Received Aug. 6, 2015; revised Jan. 5, 2016; accepted Jan. 9, 2016.

Author contributions: E.T.R., C.L.E., Q.T., M.B., S.S., and R.K. designed research; E.T.R., C.L.E., Q.T., M.B., S.S., and R.K. performed research; C.L.E., Q.T., and M.B. contributed unpublished reagents/analytic tools; E.T.R. analyzed data; E.T.R., C.L.E., Q.T., M.B., S.S., and R.K. wrote the paper.

This work was supported by the German Federal Ministry of Education and Research (BMBF; 01GQ0901, 01GQ0972, 01GQ1001A, 01GQ1403), the DFG (NeuroCure, Gottfried Wilhelm Leibniz Prize), and the ERC (NeuroBehavior grant).

The authors declare no competing financial interests.

Correspondence should be addressed to Eric T. Reifenstein, Institute for Theoretical Biology, Department of Biology, Humboldt-Universität zu Berlin, Philippstr. 13, Haus 4, 10115 Berlin, Germany. E-mail: eric.reifenstein@bccn-berlin.de.

DOI:10.1523/JNEUROSCI.2986-15.2016

Copyright (C) 2016 the authors $\quad 0270-6474 / 16 / 362283-06 \$ 15.00 / 0$ early phases of the theta oscillations, a phenomenon termed phase precession (O'Keefe and Recce, 1993).

Phase precession can be observed in the hippocampus (O'Keefe and Recce, 1993; Skaggs et al., 1996), as well as the medial entorhinal cortex (Hafting et al., 2008; Reifenstein et al., 2012, 2014) and is one of the most extensively studied examples of temporal coding in the brain (Buzsáki and Draguhn, 2004; O'Keefe and Burgess, 2005). A subpopulation of cells in layer II of the medial entorhinal cortex (mECII) shows phase precession (Hafting et al., 2008; Mizuseki et al., 2009), but it is unclear if and how phase precession is passed from the mECII to the hippocampus.

mECII contains two morphologically and electrophysiologically (Alonso and Klink, 1993; Tang et al., 2014) different types of principal cells: stellate cells, which project to the dentate gyrus, and pyramidal cells, which send output to CA1 (Varga et al., 2010; Kitamura et al., 2014; Ray et al., 2014). Differences in phase coding between the two populations would suggest that mECII passes phase precession differentially to downstream areas. Furthermore, because pyramidal and stellate cells have distinct electrophysiological properties, such as subthreshold resonance (Alonso and Klink, 1993; Engel et al., 2008), differences in temporal coding between the two populations might give insights into mechanisms underlying the generation of phase precession. 
Pyramidal and stellate cells can be reliably identified by immunohistochemistry: pyramidal cells are calbindin-positive and stellate cells are calbindin-negative (Varga et al., 2010; Tang et al., 2014). One way to directly investigate phase coding in pyramidal and stellate cells would be to perform juxtacellular recordings and labeling in freelymoving animals to directly assess the identity of the recorded cell (Tang et al., 2014). Although this approach provides unequivocal information about the cellular identity, the technique is exceedingly difficult and cannot yield sufficiently long recording durations to reliably assess phase precession. Alternatively, imaging cellular activity using fluorescent calcium indicators similarly allows for unequivocal information of cell type but does not provide the temporal resolution to measure the timing of individual spikes (Sun et al., 2015). Therefore, Tang et al. (2014) recently exploited the fact that pyramidal and stellate cells show differential locking to the local theta oscillations (Ray et al., 2014) and they developed a classifier that reliably predicts a cell's identity solely based on its spiking pattern. In the present study, we use this classifier to separate extracellularly recorded cells into putative pyramidal and putative stellate cells. This procedure allows us to perform a phase-precession analysis for sufficiently long recording durations from extracellular recordings while at the same time providing information about the identity of the cells. We are therefore able to tackle the question of whether there is a difference in phase precession between putative pyramidal and putative stellate cells in the mECII.

\section{Materials and Methods}

We analyzed extracellularly recorded data obtained from layer II of the medial entorhinal cortex in male rats. During the experiment, the animal was moving freely in a $1 \mathrm{~m}^{2}$ box. The data were previously published; see Tang et al. (2014) for details on the experimental procedures.

Isolated single units were divided into putative interneurons and putative principal cells according to spike-rate and spike-shape criteria. All recorded principal cells were assigned a label, either "putative stellate cell" (pSte) or "putative pyramidal cell" (pPyr), based on a classification procedure that was deduced from Calbindin-labeling experiments (Tang et al., 2014). The classifier was a support vector machine with a radial basis-function kernel. Two properties were used to train the classifier: the strength $S=\left|\frac{1}{N} \sum_{k=1}^{N} \exp \left(\mathrm{i} \theta_{k}\right)\right|$ of theta phase locking of a cell's spike train ( $N$ spikes) with respect to the local theta rhythm, and the cell's preferred theta phase $\varphi=\arg \left(\frac{1}{N} \sum_{k=1}^{N} \exp \left(\mathrm{i} \theta_{k}\right)\right)$ where $\theta_{k}$ depicts the theta phase of the $k$ th spike (Tang et al., 2014). The Hilbert transform was used to calculate the instantaneous phase of the theta oscillation. Spikes were excluded from the analysis if the speed of the animal was $<2 \mathrm{~cm} / \mathrm{s}$.

Only a minority of principal cells from the extracellular recordings showed clear spatial modulation (27 of 114; 21 pPyr and 6 pSte; Tang et al. (2014) shows the applied shuffling procedure). Moreover, the median
C

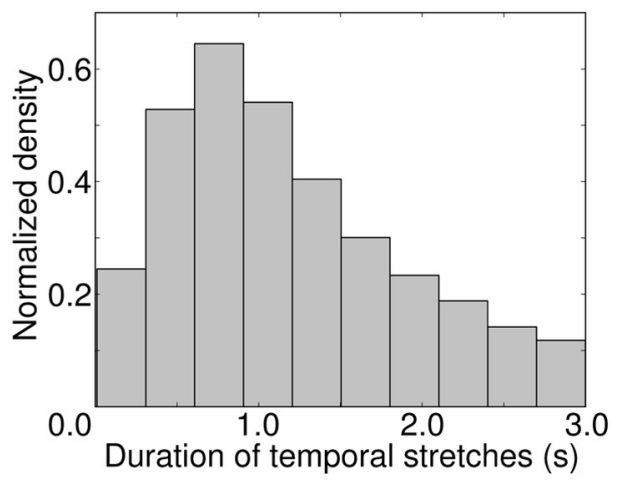

$\overline{20 \mathrm{~cm}}$

Duration of temporal stretches (s)
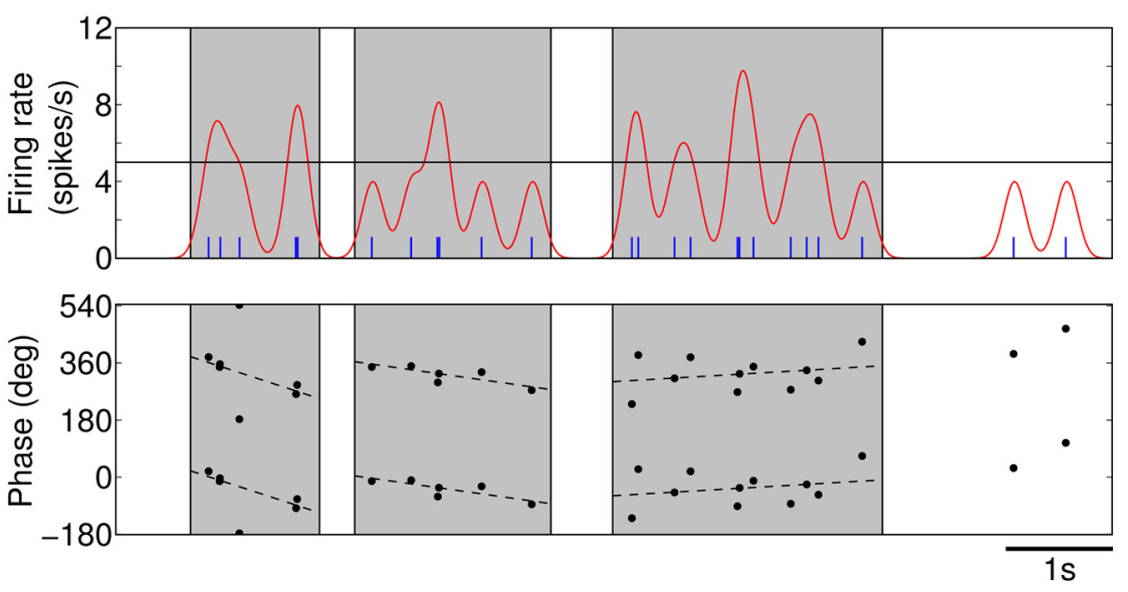

Figure 1. Detection of phase-precession patterns in temporally defined single runs of elevated firing. $A$, Spatial tuning of a putative stellate cell from $\mathrm{mEC}$ layer II. The rat moves in a $1 \mathrm{~m}^{2}$ box. Colors represent firing rates. Black lines depict temporally 列 The red curve is an estimate of the firing rate. The horizontal black line at 5 spikes/s marks the firing-rate threshold for this area, the temporal procedure can pick up additional runs (it is not limited to the spatial extent of the pooled firing fields). $\boldsymbol{C}$, Duration of temporal stretches (temporally defined single runs). The median duration is $1.39 \mathrm{~s}[1.36,1.40]$.

spatial information was low $(0.58 \mathrm{bits} / \mathrm{s}[0.49,0.72])$, and the median spatial sparseness had small values $(0.30[0.26,0.34]$; Aghajan et al., 2015). Therefore, we were not able to separate individual field traversals ("single runs") from the spatial firing fields. Instead we followed a different strategy based on the temporal structure of the recorded spike trains to identify coherent periods of elevated firing (Fig. 1; see Results for details).

For each of the single runs, the times and theta phases of all spikes were used to assess phase precession. We quantified phase precession by calculating the circular-linear correlation coefficient (Kempter et al., 2012; Reifenstein et al., 2012) and the slope of the circular-linear regression line.

To minimize errors in the classification of cells, we repeated the analysis with a "guard zone." The guard zone required cells to have a minimal distance to the decision boundary during the classification procedure. In three independent repetitions of the analysis, the width of the guard zone was chosen $0.1,0.3$, or 0.5 (Fig. $2 B$ ).

Unless stated otherwise, we used the median and a large-sample approximation for its $95 \%$ confidence interval to summarize the data as most of the data stem from skewed distributions. Accordingly, the Mann-Whitney $U$ test was used to test for statistical significance (exceptions as noted in the Results). Effects were considered statistically significant if $p<0.05$. 
A
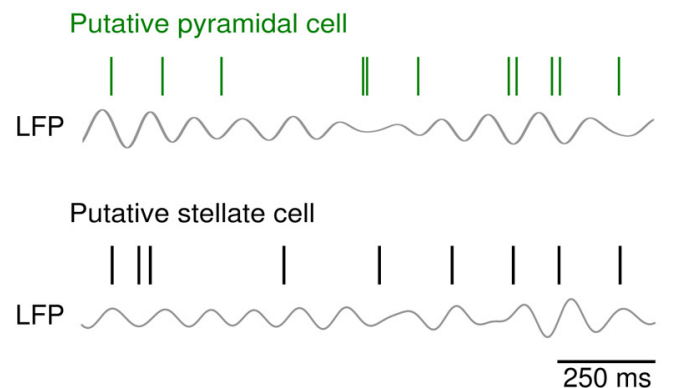

B

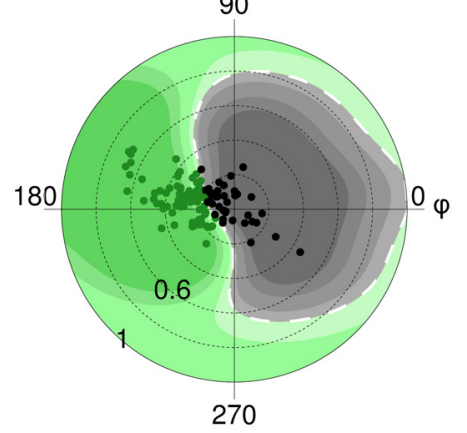

C

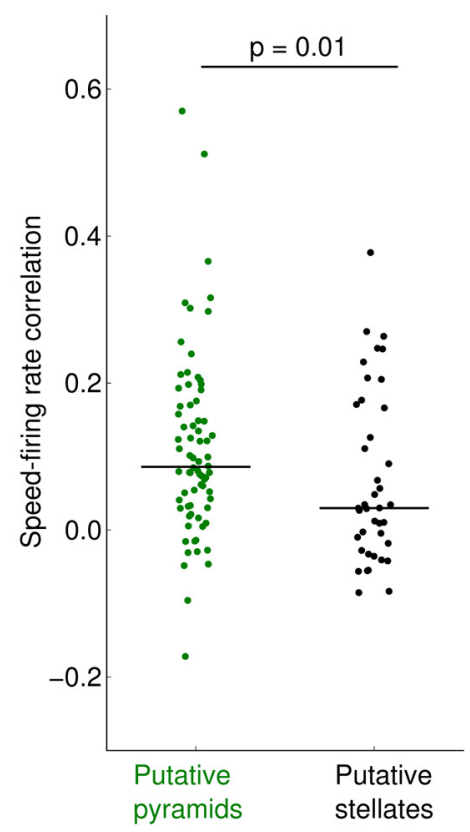

single-run phase precession correlations and slopes from the two procedures matched well (correlations: 0.01 bits Kullback-Leibler divergence; 94\% overlap between the two distributions; slopes: 0.015 bits, 93\% overlap), although the median values of the two distributions differed slightly (spatial: $-0.17[-0.18,-0.15]$, temporal: $-0.14[-0.15,-0.13], p=0.004$ for the correlations; spatial: $-76 \mathrm{deg} / \mathrm{s}$ $[-81,-72]$, temporal: $-71 \mathrm{deg} / \mathrm{s}[-74$, $-69] ; p=0.01$ for the slopes). To further quantify the similarity of the temporal and spatial selection procedures, we compared for each cell the number of spikes that were assigned to single runs by either method. We found that, on average, 97\% of in-field spikes (according to the spatial criterion) were also picked up by the temporal criterion. Additionally, the temporal criterion included $13 \%$ more spikes, which occurred at the edges of firing fields or in isolated stretches of elevated firing or in fields with low maximum rate that did not pass the detection threshold in the spatial criterion. Hence, the temporal selection of single runs generalizes the classical spatial method. In summary, we identified 15,182 single runs in a total of 114 recorded cells.

The obtained spatial distribution of the temporally defined single runs generally matched the spatial tuning of a cell (Fig. $1 A$ ), although no spatial information was used to define the single runs. The

\section{Results}

To quantify phase precession in mECII, we reanalyzed previously published data of extracellularly recorded principal cells while rats explored a $1 \mathrm{~m}^{2}$ square arena (Tang et al., 2014). The activity of the recorded cells showed a wide spectrum of weak spatial modulations (Fig. 1A). Therefore, we focused on the temporal properties of the recorded cells and did not elaborate on the spatial properties.

For irregular spatial tuning of cells as in Figure $1 A$, it was difficult to define firing fields that could be used to select individual field traversals (single runs). Instead, following Aghajan et al. (2015), we obtained candidate single runs by temporal convolution of the spike train with a Gaussian kernel of width $100 \mathrm{~ms}$, and identification of episodes above a firing-rate threshold of 5 spikes/s (Fig. $1 B$ ). As the firing rates of the cells did not vary much $(2.0$ spikes/s $[1.7,2.6], n=$ 114 ), we chose the same threshold for all cells. The candidate single runs were extended in time until the firing rate dropped $<10 \%$ of the single run's peak firing rate and remained $<10 \%$ for $>250 \mathrm{~ms}(\sim 2$ theta cycles) before and after the run. The latter criterion takes into account that the firing activity might skip theta cycles. Finally, the individual runs were required to have a minimal duration of $300 \mathrm{~ms}$ and to include at least four spikes (Fig. 1B).

To justify the choice of parameters to select single runs based on their temporal structure, we applied this method also to data from previous studies (Sargolini et al., 2006; Reifenstein et al., 2014) in which clear firing fields could be identified. Thus, we were able to apply both the "classical" spatial selection and the proposed temporal selection of single runs. The distributions of temporal stretches of spiking activity had a median duration of 1.39 s $([1.36,1.40]$ s; Fig. $1 C)$.

For each single run, phase precession was quantified by circular-linear regression (Kempter et al., 2012). Negative slopes indicated phase precession, but also single runs with positive slopes were observed (Fig. 1B). The obtained slopes $(-54 \mathrm{deg} / \mathrm{s}$ $[-57,-51])$ and correlations $(-0.104[-0.112,-0.095])$ were slightly smaller than for the dataset used by Reifenstein et al. (2014) in which cells from all cortical layers were analyzed (data from Sargolini et al., 2006; slopes: $-71 \mathrm{deg} / \mathrm{s}$ [ -74, -69]; correlation: $-0.14[-0.15,-0.13]$, reanalysis using the temporal selection of single runs). Yet, the distributions of correlations overlapped strongly for the two datasets (94\%), indicating that they match the ranges of values in Reifenstein et al. (2014). The difference in median values of slopes might be explained by the shorter single runs in the Sargolini dataset $(0.97 \mathrm{~s}[0.96,0.98]$ vs $1.39 \mathrm{~s}[1.36,1.40]$ in our data, $\left.p<10^{-50}\right)$. Shorter runs suggest that the average field sizes were smaller in the Sargolini data than in our data. As a consequence, the slopes in the Sargolini dataset would be steeper because smaller fields have steeper slopes (Reifenstein et al., 2014).

To quantify phase precession for pyramidal and stellate cells in layer II separately, we distinguished two classes of principal cells in our analysis. Tang et al. (2014) and Ray et al. (2014) showed that putative pyramidal cells tended to fire spikes around the trough of the local theta oscillation; on the other hand, the spiking activity of putative stellate cells showed a weak preference for the peak of theta (Fig. 2A). Based on this finding, we 
were able to separate extracellularly recorded units into pPyr and pSte (Fig. 2B). In a total of 74 putative pyramidal cells and 40 putative stellate cells (an example of the activity a putative stellate cell was shown in Fig. 1A), we identified 11,684 and 3,498 single runs, respectively. Although the two populations strongly differed in phase-locking strength (pPyr: $0.33 \pm 0.02$, pSte: $0.15 \pm$ $0.01 ; p=8 \times 10^{-12}, t$ test $)$, they showed similar overall firing rates (pPyr: 2.0 spikes/s $[1.5,2.9]$; pSte: 2.1 spikes/s $[1.4,2.7] ; p=$ 0.19 ) and similar bursting indexes (proportion of interspike intervals smaller than $0.15 \mathrm{~s}$, pPyr: $0.50[0.44,0.58]$; pSte: 0.47 $[0.39,0.55] ; p=0.18)$.

To gain more confidence in the classification procedure, we investigated the relationship between movement speed and firing rate for putative pyramidal cells and putative stellate cells. Sun et al. (2015) showed that pyramids have a stronger speed modulation of the firing rate. We tested this feature by calculating the correlation between the speed of the animal and the temporal fluctuations of the firing rate for each cell (Fig. $1 B$, see red curve for an example of firing-rate fluctuations). The firing rate and the speed were significantly correlated for both classes of cells, but the average correlation was significantly stronger for putative pyramidal cells (pPyr: $r=0.09$ [0.07, 0.12]; pSte: $r=0.03$ $[-0.002,0.09] ; p=0.01$; Fig. $2 C)$, confirming the classification proposed by Tang et al. (2014).

Interestingly, putative stellate cells displayed steeper phase precession than putative pyramidal cells (pPyr: $-47 \mathrm{deg} / \mathrm{s}[-50,-44]$; pSte: $-81 \mathrm{deg} / \mathrm{s}[-87,-74] ; p=10^{-13}$; Fig. $\left.3 A, B\right)$. To assess the size of the effect (difference of the median phase-precession slopes), we randomly assigned cell identities ("pPyr" or "pSte") to the single runs and calculated the effect size for each realization. We found that the measured effect size is larger than expected by chance in $>99.8 \%$ of all cases (corresponds to $p=0.013$ ).

To exclude sample size effects, we randomly downsampled the large number of single runs from the putative pyramidal cells ( $n=11,684)$ to match the smaller number of runs from putative stellate cells $(n=3,498)$. In all 1000 repetitions of the downsampling, we found significantly shallower phase precession in the putative pyramidal cells as compared with the putative stellate cells ( $p=10^{-6}$ for all 1000 repetitions).

The correlation between time and theta phase was similar in putative pyramidal cells and putative stellate cells (pPyr: -0.10 $[-0.11,-0.09]$; pSte: $-0.11[-0.13,-0.09], p=0.1)$. The steeper phase precession in putative stellate cells was accompanied by a larger phase range (pPyr: $110 \mathrm{deg}[106,113]$; pSte: $169^{\circ}$ $[156,178] ; p=2 \times 10^{-6}$; Fig. $3 C$ ), because the duration of spike sequences did not differ for the two populations (pPyr: $1.40 \mathrm{~s}$ [1.37, 1.42]; pSte: 1.31 s [1.29, 1.37]; $p=0.12$; Fig. 3D).

To minimize an erroneous classification of cells that were close to the decision boundary, we also implemented a guard zone: cells were required to have a minimal distance to the decision boundary during the classification procedure (Fig. 2B). For all tested minimal distances $(0.1,0.3$, and 0.5$)$, putative stellate cells exhibited significantly steeper phase precession than putative pyramidal cells $\left(p=3 \times 10^{-14}\right.$, minimal distance $=0.1 ; p=$ $6 \times 10^{-12}$, minimal distance $=0.3 ; p=4 \times 10^{-5}$, minimal distance $=0.5$ ).

The classification procedure of cells depended on the theta locking of spikes. Could this procedure bias the phase precession of the two populations? For example, perfect phase locking (phase-locking strength $=1$ ) would impede phase precession. We measured the average phase-precession slope and the phaselocking strength for each cell. We found no correlation $(r=0.14$, $p=0.15$; Fig. $3 E$ ) between the two measures. Also for the two
A
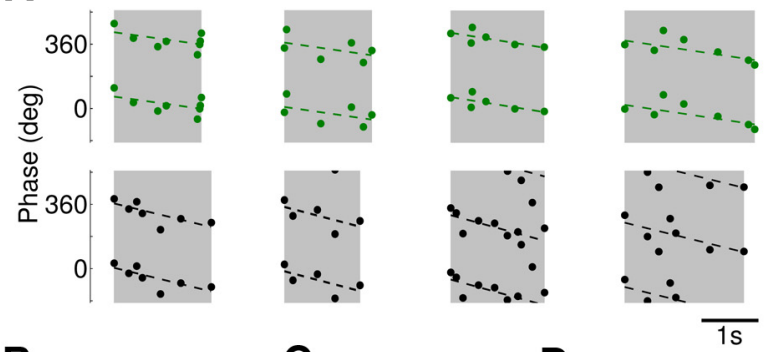

B C

D

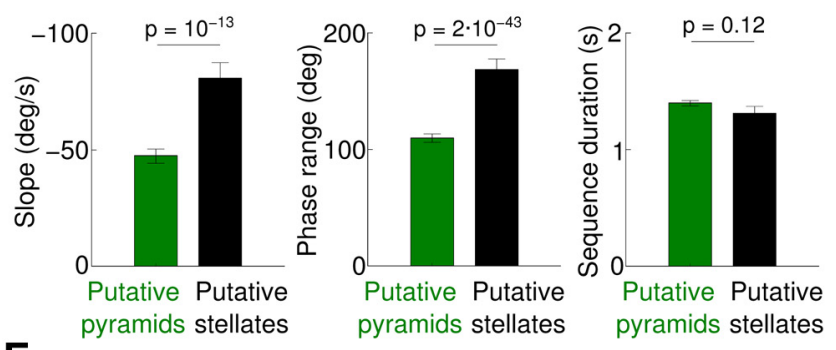

E

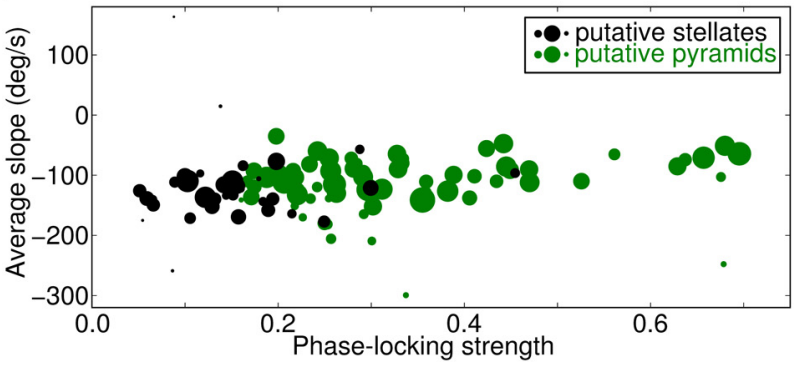

Figure 3. Phase precession was steeper in putative stellate cells. $\boldsymbol{A}$, Examples of single-run phase precession for a putative pyramidal cell (top) and a putative stellate cell (bottom). Green and black dots represent the spikes of the putative pyramidal and stellate cells, respectively. Dashed lines depict circular-linear fits. $\boldsymbol{B}$, Phase-precession slopes were significantly steeper in putative stellate cells. $\boldsymbol{C}$, Single-run phase ranges were significantly larger in putative stellate cells, consistent with the larger slope of phase precession. $\boldsymbol{D}$, The duration of single runs is statistically the same for the two populations. In $\boldsymbol{B}-\boldsymbol{D}$, error bars depict the $95 \%$ confidence interval of the median. $\boldsymbol{E}$, Phase-locking strength and phase-precession slopes were not correlated in a cell-by-cell analysis. Green and black dots represent putative pyramidal cells and putative stellate cells, respectively. The area of each dot is proportional to the number of runs that the corresponding cell contributed to the analysis.

populations separately, phase-precession slope and phaselocking strength did not correlate (pPyr: $r=0.19, p=0.11$; pSte: $r=0.05, p=0.77)$. Together, these results indicate that-within the physiological range of phase-locking strength $<0.7$ (Fig. $3 E)$ - phase precession and phase locking can coexist.

\section{Discussion}

We found that putative stellate cells display steeper phase precession than putative pyramidal cells, which was accompanied by larger phase ranges. This is the first account of phase precession in different cell types within mECII. Hafting et al. (2008) and Mizuseki et al. (2009) showed entorhinal phase precession in extracellular recordings of unidentified cells in layer II, but did not relate the phase precession to pyramidal or stellate cells. Newman and Hasselmo (2014) investigated phase-precession of grid cells recorded in the superficial layers of $\mathrm{mEC}$ (both layers II and III) based on pooled-run data and found larger phase-precession correlations in trough-locking grid cells as compared with peaklocking grid cells. In contrast to this, we quantified phase precession in single runs of principal cells from only mECII and could 
not find differences in the distributions of phase-precession correlations. Furthermore, Newman and Hasselmo (2014) suggested that trough-locking cells might be stellate cells, based on the stellate cells' tendency to fire in bursts during recordings in slices. We did not find differences in the bursting behavior of the putative pyramidal and putative stellate cells.

Newman and Hasselmo (2014) reported pooled-data phaseprecession correlations in the range of -0.05 . Because of the broader scatter of data points, pooled-data correlations are generally expected to be lower than single-run correlations (Schmidt et al., 2009). Therefore it is not surprising that we found twofold stronger correlations in our single-run analysis (in the range of -0.1 ). We argue that the animal needs to process information online and does not have the opportunity to pool over trials. Apart from that, functional hypotheses about temporal coding (Mehta et al., 2002) or sequence learning (Hasselmo and Eichenbaum, 2005) are usually discussed for phase precession in individual runs. Hence, it is appropriate to also analyze phase precession in single runs.

Aghajan et al. (2015) measured phase-precession correlations in temporally defined single runs and found correlations of around 0.2 . The difference to our result might be explained by three facts: Aghajan et al. (2015) (1) recorded in CA1; (2) used a linear-circular correlation which is based on a sinusoidal model, in contrast to the circular-linear correlation we used (Kempter et al., 2012); and (3) restricted their analysis to significantly phaseprecessing cells, whereas we included all cells in the analysis. The slopes of phase precession in our dataset were shallower than in the single-run analysis by Reifenstein et al. (2014) of mECII grid cells recorded by Sargolini et al. (2006). In the latter dataset, temporally defined single runs are shorter than in our data, suggesting that field sizes are smaller. Smaller field sizes were shown to correspond to steeper phase-precession slopes (Reifenstein et al., 2014). Therefore, it is likely that differences between our results and the findings by Reifenstein et al. (2014) are because of different field sizes in the two datasets.

It is presently unclear whether grid cells in mECII are predominantly pyramidal cells (Tang et al., 2014), stellate cells (Domnisoru et al., 2013), or if they show no preference for either cell type (Sun et al., 2015). Tang et al. (2014) found that in mECII calbindin-positive putative pyramidal cells on average showed higher grid scores than calbindin-negative putative stellate cells. Conversely, putative stellate cells generally showed higher border scores. We add to the picture that putative stellate cells exhibit steeper phase precession than putative pyramidal cells. It should be noted, however, that all conclusions rest on the assumption that the classifier of Tang et al. (2014) works correctly. To control for problems arising from our classification procedure, we performed three checks: first, we repeated the analysis using a variable guard zone, and found that our results did not depend on the guard zone width. Second, we replicated the recent finding from Sun et al. (2015) and found that the activity of putative pyramidal cells is more modulated by the animal's running speed than the activity of putative stellate cells. Third, we found no correlation between phase-precession slope and the phase-locking strength. This suggests that our results are not just an artifact of the known difference in phase-locking strength between pyramidal and stellate cells (Ray et al., 2014; Tang et al., 2014), but a real difference in the phase coding of the two cell populations.

Phase precession might be used to compress behavioral sequences to the time scale of synaptic plasticity (Skaggs et al., 1996). In this framework, a steeper phase-precession slope implies that the spikes of different cells in the compressed sequence are temporally more separated, which might be beneficial to retain the order of the sequence under the influence of temporal noise. On the other hand, a clearer temporal separation of the spikes from different cells poses a limit on the number of cells participating in the sequence.

Our results suggest that stellate cells send steeply phaseprecessing output to the dentate gyrus, whereas the shallower precessing mECII pyramidal cells project to CA1 (Varga et al., 2010; Kitamura et al., 2014). Phase precession might thus be differentially inherited by different subregions of the hippocampal-entorhinal loop (Jaramillo et al., 2014; Schlesiger et al., 2015).

To conclude, our data indicate that even within the same layer of mEC principal cells can show different phase-precession patterns. This result compares with other observations which indicate that different groups of CA1 pyramidal cells can exhibit distinct temporal discharge patterns (Mizuseki et al., 2011). Also spiking activity of hippocampal interneurons is highly cell-typespecific (Klausberger and Somogyi, 2008). Hence, we argue that cell-type-specific temporal coding is a widespread phenomenon throughout the hippocampal formation. Future studies might aim to further investigate where phase precession originates and whether or how this temporal code is passed on between different subregions.

\section{References}

Aghajan ZM, Acharya L, Moore JJ, Cushman JD, Vuong C, Mehta MR (2015) Impaired spatial selectivity and intact phase precession in twodimensional virtual reality. Nat Neurosci 18:121-128. CrossRef Medline

Alonso A, Klink R (1993) Differential electroresponsiveness of stellate and pyramidal-like cells of medial entorhinal cortex layer II. J Neurophysiol 70:128-143. Medline

Buzsáki G, Draguhn A (2004) Neuronal oscillations in cortical networks. Science 304:1926-1929. CrossRef Medline

Domnisoru C, Kinkhabwala AA, Tank DW (2013) Membrane potential dynamics of grid cells. Nature 495:199-204. CrossRef Medline

Engel TA, Schimansky-Geier L, Herz AV, Schreiber S, Erchova I (2008) Subthreshold membrane-potential resonances shape spike-train patterns in the entorhinal cortex. J Neurophysiol 100:1576-1589. CrossRef Medline

Hafting T, Fyhn M, Bonnevie T, Moser MB, Moser EI (2008) Hippocampus-independent phase precession in entorhinal grid cells. Nature 453:1248-1252. CrossRef Medline

Hasselmo ME, Eichenbaum H (2005) Hippocampal mechanisms for the context-dependent retrieval of episodes. Neural Netw 18:1172-1190. CrossRef Medline

Jaramillo J, Schmidt R, Kempter R (2014) Modeling inheritance of phase precession in the hippocampal formation. J Neurosci 34:7715-7731. CrossRef Medline

Kempter R, Leibold C, Buzsáki G, Diba K, Schmidt R (2012) Quantifying circular-linear associations: hippocampal phase precession. J Neurosci Methods 207:113-124. CrossRef Medline

Kitamura T, Pignatelli M, Suh J, Kohara K, Yoshiki A, Abe K, Tonegawa S (2014) Island cells control temporal association memory. Science 343: 896-901. CrossRef Medline

Klausberger T, Somogyi P (2008) Neuronal diversity and temporal dynamics: the unity of hippocampal circuit operations. Science 321:53-57. CrossRef Medline

Mehta MR, Lee AK, Wilson MA (2002) Role of experience and oscillations in transforming a rate code into a temporal code. Nature 417:741-746. CrossRef Medline

Mizuseki K, Sirota A, Pastalkova E, Buzsáki G (2009) Theta oscillations provide temporal windows for local circuit computation in the entorhinalhippocampal loop. Neuron 64:267-280. CrossRef Medline

Mizuseki K, Diba K, Pastalkova E, Buzsáki G (2011) Hippocampal CA1 pyramidal cells form functionally distinct sublayers. Nat Neurosci 14: 1174-1181. CrossRef Medline

Newman EL, Hasselmo ME (2014) Grid cell firing properties vary as a function of theta phase locking preferences in the rat medial entorhinal cortex. Front Syst Neurosci 8:193. Medline 
O’Keefe J, Burgess N (2005) Dual phase and rate coding in hippocampal place cells: theoretical significance and relationship to entorhinal grid cells. Hippocampus 15:853-866. CrossRef Medline

O'Keefe J, Recce ML (1993) Phase relationship between hippocampal place units and the EEG theta rhythm. Hippocampus 3:317-330. CrossRef Medline

Ray S, Naumann R, Burgalossi A, Tang Q, Schmidt H, Brecht M (2014) Grid-layout and theta-modulation of layer 2 pyramidal neurons in medial entorhinal cortex. Science 343:891-896. CrossRef Medline

Reifenstein ET, Kempter R, Schreiber S, Stemmler MB, Herz AV (2012) Grid cells in rat entorhinal cortex encode physical space with independent firing fields and phase precession at the single-trial level. Proc Natl Acad Sci U S A 109:6301-6306. CrossRef Medline

Reifenstein E, Stemmler M, Herz AV, Kempter R, Schreiber S (2014) Movement dependence and layer specificity of entorhinal phase precession in two-dimensional environments. PLoS One 9:e100638. CrossRef Medline

Sargolini F, Fyhn M, Hafting T, McNaughton BL, Witter MP, Moser MB, Moser EI (2006) Conjunctive representation of position, direction, and velocity in entorhinal cortex. Science 312:758-762. CrossRef Medline

Schlesiger MI, Cannova CC, Boublil BL, Hales JB, Mankin EA, Brandon MP,
Leutgeb JK, Leibold C, Leutgeb S (2015) The medial entorhinal cortex is necessary for temporal organization of hippocampal neuronal activity. Nat Neurosci 18:1123-1132. CrossRef Medline

Schmidt R, Diba K, Leibold C, Schmitz D, Buzsáki G, Kempter R (2009) Single-trial phase precession in the hippocampus. J Neurosci 29: 13232-13241. CrossRef Medline

Skaggs WE, McNaughton BL, Wilson MA, Barnes CA (1996) Theta phase precession in hippocampal neuronal populations and the compression of temporal sequences. Hippocampus 6:149-172. CrossRef Medline

Sun C, Kitamura T, Yamamoto J, Martin J, Pignatelli M, Kitch L, Schnitzer MJ, Tonegawa S (2015) Distinct speed dependence of entorhinal island and ocean cells, including respective grid cells. Proc Natl Acad Sci U S A 112:9466-9471. CrossRef Medline

Tang Q, Burgalossi A, Ebbesen CL, Ray S, Naumann R, Schmidt H, Spicher D, Brecht M (2014) Pyramidal and stellate cell specificity of grid and border representations in layer 2 of medial entorhinal cortex. Neuron 84: 1191-1197. CrossRef Medline

Varga C, Lee SY, Soltesz I (2010) Target-selective GABAergic control of entorhinal cortex output. Nat Neurosci 13:822-824. CrossRef Medline 